Article

\title{
Adaptive Sliding Mode PID Control for Underwater Manipulator Based on Legendre Polynomial Function Approximation and Its Experimental Evaluation
}

\author{
Chao Yang ${ }^{1,2, *}$, Feng Yao ${ }^{1}{ }^{1}$, Mingjun Zhang ${ }^{1}$, Zhiqiang Zhang ${ }^{2}$, Zhenzhen $\mathrm{Wu}^{3}$ and \\ Peijian Dan ${ }^{2}$ \\ 1 College of Mechanical and Electrical Engineering, Harbin Engineering University, Nangang District, \\ Harbin 150001, China; yaofeng@hrbeu.edu.cn (F.Y.); zhangmingjun@hrbeu.edu.cn (M.Z.) \\ 2 Institute of Materials, China Academy of Engineering Physics, Huafeng Village, Jiangyou, Mianyang 621907, \\ Sichuan, China; chaucer123456@126.com (Z.Z.); lovezhen230026@126.com (P.D.) \\ 3 Finance \& Trade, Chongqing Vocational Institute of Engineering, Jiangjin 402260, Chongqing, China; \\ loveavrilsong@163.com \\ * Correspondence: a0232072590@126.com; Tel.: +86-451-8251-9704
}

Received: 4 February 2020; Accepted: 27 February 2020; Published: 3 March 2020

\begin{abstract}
The joint control problem of the underwater manipulator is addressed in this paper, under the influence of uncertainty factors such as model uncertainty, external disturbance, and manipulator joint lag. In general, for the uncertainty factors, it is usually approximated online, but it is difficult to select a reasonable value for the approximation error boundary, too conservative estimated values would cause chattering problem easily. And the influence of joint lag on the manipulator control should be considered in actual work. Unlike most previous control method, in this paper, the function approximation technique (FAT), which uses the Legendre polynomial, is adopted to approximate the uncertainty factors online. Then, based on the proportion integral differential (PID) sliding manifold with the integral and differential of tracking error, a sliding model PID controller is designed to speed up the response and reduce the effects of joint lag. For the error boundary, the adaptive law is proposed, and it will reduce chattering of the control quantity under the steady state of the system. It was proved that the joint error of the control system is uniformly asymptotic convergence through the stability analysis. Finally, the effectiveness of the proposed approach is demonstrated with pool comparison experiments of the underwater manipulator installed in the autonomous underwater vehicles (AUVs).
\end{abstract}

Keywords: underwater manipulator; sliding mode control; function approximation technique; joint lag; error boundary; uncertainty estimation

\section{Introduction}

In the complex sea, autonomous underwater vehicles (AUVs) play an irreplaceable role for various missions, such as exploration of marine resource, environmental monitoring, seabed topographic survey, and target search [1,2]. In recent years, with the deepening of marine development, simple detection and target search cannot meet the operational requirements. However, the underwater vehicle-manipulator system (UVMS), which consists of underwater vehicle and manipulators, and its operating technology have been rapidly developed and widely used in the fields of scientific research and ocean systems engineering for performing interactive tasks, such as opening and closing of valves, cutting in coordination, and so on [3,4]. An AUV when equipped with a manipulator becomes a kinematically redundant system, it has more degrees of freedom (DOF) than is required to perform a task in its operational space. In TRIDENT EU FP7 project, an underwater electric manipulator was 
developed and equipped on multipurpose intervention-AUV [4]. An underwater electric manipulator (MARIS 7080, 7 DOF manipulator) was equipped on semi-autonomous underwater vehicle for the intervention mission (SAUVIM) [5].

In UVMS, the underwater manipulator is a key component, and its joint control accuracy directly determines the operating accuracy of the UVMS. At present, underwater manipulators are mostly control objects with joint redundancy, and the accuracy mainly depends on the control performance of joints [6]. However, due to the nonlinear, time-variation of dynamic properties and other factors (i.e., external disturbances such as ocean current disturbance) $[7,8]$, the system dynamic model has uncertainties, which will affect the joint control of the manipulator based on the dynamic model. Besides, for most hydraulic manipulators, the joint control performance is also affected by joint lag $[6,9,10]$, which means that the joint lag will affect the control accuracy of manipulator joint. In conclusion, under the influence of the above factors, it has great research significance and practical value to develop the joint control technology of underwater manipulators for improving the accuracy and efficiency of underwater operation.

Currently, many methods have achieved high control accuracy, which uses the online approximation to deal with the uncertainty factors, and determine the controller and adaptive law of parameter through stability analysis [11,12]. Among them, in view of the nonlinear and uncertain identification ability [11-16], neural network and fuzzy control has been used in the joint control of manipulator. In [13], a feedforward neural network is employed to learn the parametric uncertainties, existing in the dynamical model of the robot manipulator, and the influence of weight and bias weight of the two-layer neural network are considered, but this increases the difficulty of designing the weight adjustment law, and an excellent neural network has relatively more control parameters, and selection of initial values of neural network parameters will increase the difficulty of the controller design and the complexity of the stability analysis, and also the adaptive adjustment of control parameters will increase the load on the hardware system. In [14], a novel robust decentralized control of electrically driven robot manipulators by adaptive fuzzy estimation and compensation of uncertainty, and in the fuzzy controllers, it uses expert's knowledge, the trial and error method, or an optimization algorithm such as particle swarm optimization (PSO) to design the fuzzy rules, and the design process may be more complicated and not online.

Recently, some researchers have proposed the regressor-free control of manipulators based on function approximation technique (FAT) [17-20], in which the uncertainty factors have been estimated or approximated using the Taylor function expansion [18], Fourier series [19], or Legendre polynomial $[17,20]$. Compared with the neural network and fuzzy control, its control strategy is simpler, and the influence of the initial value selection on the controller is reduced since the tuning parameters are less. In [18], it uses the first-order Taylor series expansion to propose an adaptive estimator of uncertainty for the robust control of electrically driven manipulators, to improve the computational efficiency and ease of implementation, the higher-order terms of Taylor series expansion are ignored. Instead, the higher-order terms may cause approximation errors and the estimator performance may be reduced. However, for the Fourier series, due to the characteristic of the basis function of the Fourier approximation, its approximation effect on the periodic function is the most obvious. Furthermore, in [17], it uses the Legendre polynomial to approximate the uncertainty factors online, and the Legendre coefficients can be tuned using the adaptive laws derived from the stability analysis. However, it is difficult to select a reasonable value for the error boundary in [17], too conservative values would cause high-frequency chattering of the system, and too low would increase tracking error. Furthermore, for the underwater manipulator of this article (as shown in [9]), under the drive of hydraulic system, there is joint lag in the joint control of the manipulator. However, the above control methods, such as [17], do not consider the influence of joint lag on the trajectory tracking control.

For the above considerations, this paper proposes an adaptive sliding mode proportion integral differential (PID) control method for underwater manipulator based on Legendre polynomial FAT. In our work, a sliding model PID controller is designed to accelerate the system response and reduce 
joint lag. The adaptive law is used to estimate the error boundary, and reduce the chattering of the system. Furthermore, according to the analysis of Lyapunov stability, it is demonstrated that the tracking error can be uniformly asymptotically converged. Based on the pool experiments of the joint step response and trajectory tracking, the effectiveness of the proposed method is verified by comparing the joint error and control quantity of the proposed method and the comparison method in [17].

This paper is organized, as follows. Section 2 describes the joint control problem of the underwater manipulator, and gives the dynamics model and its uncertainties. In Section 3, the adaptive sliding mode PID control method is studied based on Legendre polynomial FAT, and contains the stability analysis. Then in Section 4, the effectiveness of the proposed method is verified by pool experiments. Finally, we make a brief conclusion in Section 5.

\section{Problem Formulation}

In this section, under the influence of uncertainty factors such as model uncertainty and external disturbance, the establishment of the dynamics model of the manipulator is mainly explained. According to the $[13,21,22]$, the dynamics model containing the above uncertainty factors is as follows:

$$
H(q) \ddot{q}+C(q, \dot{q}) \dot{q}+G(q)=\tau+F
$$

where, $q, \dot{q}, \ddot{q} \in R^{n}$ are the position, velocity and acceleration vectors for each joint. $H(q) \in R^{n \times n}$ is the inertia matrix including extra mass, the matrix $C(q, \dot{q}) \in R^{n \times n}$ groups is the centripetal and Coriolis forces, and $G(q) \in R^{n \times n}$ is the gravity matrix vector. $F$ is the external disturbances (including water flow and other external disturbances.). $\tau$ is the driving torque and $n$ is the number of degrees of freedom.

According to the [23], the Equation (1) is transformed into a state space equation form for description:

$$
\ddot{\eta}(t)=F(\eta, t)+G(\eta, t) U+D(t)
$$

where, $U=\left[\tau_{1}, \tau_{2} \ldots \tau_{n}\right]^{\mathrm{T}}$ is the control vector for each joint. $D(t)$ is the external disturbances after conversion. $F(\eta, t)$ and $G(\eta, t)$ are the matrix vectors. In the Equation $(2), F(\eta, t), G(\eta, t)$, and $D(t)$ are represented by the Equation (3):

$$
\begin{gathered}
F(\eta, t)=-H(q)^{-1}[C(q, \dot{q}) \dot{q}+G(q)] \\
G(\eta, t)=-\lambda H(q)^{-1} \\
D(t)=-H(q)^{-1} F
\end{gathered}
$$

In practice work, there exists uncertainty factors in the dynamics model, and the control of manipulator is affected by external disturbances. Therefore, the nonlinear matrix vectors $F(\eta, t), G(\eta, t)$, and $D(t)$ in Equation (2), which contains uncertainties, cannot be obtained exactly. So, we convert Equation (2) to Equation (4).

$$
\begin{gathered}
U=\ddot{\eta}(t)+H(\eta, t) \\
H(\eta, t)=\left[G(\eta, t)^{-1}-I\right] \ddot{\eta}(t)-G(\eta, t)^{-1}[F(\eta, t)+D(t)]
\end{gathered}
$$

where, the nonlinear term $H(\eta, t)$ contains all uncertainty factors, and cannot accurately be described. For this reason, we will approximate $H(\eta, t)$ online.

In summary, the control objective of this paper is to design an adaptive controller $U$ of the joints control of underwater manipulator, which can effectively overcome the influence of uncertainties such as model uncertainty, external disturbance. It aims to ensure the boundedness of the closed-loop system, make the tracking error uniformly asymptotic converge. 


\section{Controller Design Based on Legendre Polynomial FAT}

This section addresses the adaptive sliding mode PID control method for the underwater manipulator based on Legendre polynomial FAT, and then designs its adaptive sliding mode PID controller. Moreover, based on the analysis of Lyapunov stability theory, it is theoretically proved that the joint error is uniformly asymptotic convergence.

\subsection{Uncertainty Factors Function Approximation Based on the Legendre Polynomial}

This subsection describes the function approximation process of uncertainty factors $H(\eta, t)$ based on Legendre polynomial. Compared with the typical neural network and fuzzy control approximation method $[14,16]$, the approximation strategy using the Legendre polynomial FAT is simpler and needs less computational power. Since the selection of the initial value of the parameter is reduced, it can be effectively reduce the difficulty of stability analysis.

Next, the function approximation process based on the Legendre polynomial was described.

\subsubsection{Legendre Polynomial Function Approximation}

Suppose that $V$ is the space of all real-valued continuous-time functions. According to the $[17,20,24]$, a nonlinear function $h(x)$, which defined on the interval $\left[x_{1} x_{2}\right]$ in $V$, can be represented as the function approximation of Equation (6a).

$$
h(x)=\sum_{i=0}^{m} \alpha_{i} \varphi_{i}(x)+\varepsilon_{m}(x)
$$

where, $\varphi_{0}(x), \varphi_{1}(x) \ldots . . \varphi_{\mathrm{m}}(x)$ is a $m$-th Legendre polynomial, and satisfies the orthogonal basis. $\varepsilon_{\mathrm{m}}(x)$ is the approximation error, and bounded [20]. $\alpha_{i}$ is approximation coefficient, as follows.

$$
\begin{gathered}
\alpha_{i}=\frac{1}{A_{i}} \int_{x_{1}}^{x_{2}} h(x) \varphi_{i}(x) d x i=0,1, \ldots \ldots m \\
\int_{x_{1}}^{x_{2}} \varphi_{i}(x) \varphi_{j}(x) d x=\left\{\begin{array}{c}
0 i \neq j \\
A_{i} i=j
\end{array}\right.
\end{gathered}
$$

he Legendre polynomial requires that the nonlinear $h(x)$ must be defined on the interval [-11] (i.e., [ $x_{1}$ $\left.x_{2}\right]$ is [-11]). Therefore, $\varphi_{i}(x)$ in Equation (6) can be defined by Equation (7).

$$
\begin{gathered}
\varphi_{0}(x)=1, \varphi_{1}(x)=x \\
(i+1) \varphi_{i+1}(x)=(2 i+1) x \varphi_{i}(x)-i \varphi_{i-1}(x)
\end{gathered}
$$

Thus, the $h(x)$ defined on the [-11] can be approximated by Legendre polynomial in the form of (6), which the coefficients $\alpha_{i}(i=0,1, \ldots, m)$ are calculated by Equations (3) and (4), and the $\varphi_{i}(x)(i=0$, $1, \ldots, m)$ are given by Equation (7). Finally, the transformation of the matrix expression of the Equation (6) can be obtained by the Equations (8) and (9).

$$
\begin{gathered}
h(x)_{L P}=P^{T} \varphi(x)+\varepsilon_{m}(x) \\
P=\left[\alpha_{0} \alpha_{1} \ldots \ldots \ldots \ldots \alpha_{m}\right]^{T} \\
\varphi(x)=\left[\varphi_{0}(x) \varphi_{1}(x) \ldots \ldots \ldots . \varphi_{m}(x)\right]^{T}
\end{gathered}
$$

where, $h(x)_{L P}$ is the approximation function after transformed; $\alpha_{i}$ is vectorized to obtain the approximation coefficient $P$.

Note 1. In Equation (9), $P$ cannot be calculated by the Equations (3) and (4), since $h(x)_{L P}$ is unknown. For the controller of this paper, this parameter is adjusted online by the adaptive control law.

Note 2. In [20], another important issue about function approximation may be the fact that the $\varphi_{i}(x)$ are mutually orthogonal just on the interval [-11]. Out of this interval, the functions $\varphi_{i}(x)$ may 
not be mutually orthogonal. However, for the $H(\eta, t)$, which is a nonlinear function based on the motion state and time, it is impossible to be limited to $[-1,1]$. For this problem, the author makes $x=$ $\sin (\omega t)$ in the Equation (7), $\omega$ is a predefined constant.

Then, combining the Equations (7) and (9b), the $\varphi(x)$ can be expressed as:

$$
\varphi(x)=\left[1, x, \frac{1}{2}\left(3 x^{2}-1\right), \frac{1}{2}\left(5 x^{3}-3 x\right), \frac{1}{8}\left(35 x^{4}-30 x^{2}+3\right), \ldots \ldots\right]
$$

where $x=\sin (\omega t) \in[-1,1]$ satisfies the interval requirement.

\subsubsection{Online Approximation of $H(\eta, t)$}

Based on the above analysis, the online approximation of $H(\eta, t)$ was performed. In the theoretical approximation of uncertainty, the online approximation of $H(\eta, t)$ was obtained as shown in Equation (11).

$$
H(\eta, t)=P^{T} \varphi(x)+\varepsilon_{m}(x)
$$

However, in actual online approximation, the actual approximation value is $\hat{H}(\eta, t)$, as follows

$$
\hat{H}=\hat{P}^{T} \varphi(x)
$$

where, $\hat{P}$ and $\hat{H}$ are the estimation of $P$ and $H$ respectively, $\widetilde{P}=P-\hat{P}$. The $\varepsilon_{m}(x)$ is bounded, and defines $\left|\varepsilon_{m}(x)\right| \leq \rho$, and $\rho$ is the error-boundary, which is derived from the error-boundary control law.

\subsection{Adaptive Sliding Mode PID Controller Design}

\subsubsection{Sliding Mode Manifold Design}

The sliding mode control has been widely used in nonlinear systems, due to its robustness to modeling uncertainty and external disturbances $[16,25,26]$. However, for the sliding mode control, the conventional linear sliding mode manifold can be used to eliminate the steady-state error and guarantee the asymptotic stability of system errors, but the tracking error cannot converge to zero in finite time, so it cannot solve the motion lag caused by the joint lag. To ensure the quick convergence of the joint errors and reduce the motion lag caused by the joint lag, this paper designed a PID sliding mode manifold with the integral and differential of tracking error, shown in Equation (13).

$$
\begin{gathered}
s=k_{p} e(t)+k_{i} \int e(t) d t+k_{d} \dot{e}(t) \\
e(t)=\eta_{d}-\eta
\end{gathered}
$$

where, $k_{\mathrm{p}}, k_{\mathrm{i}}$, and $k_{\mathrm{d}}$ are the positive constant, and $k_{\mathrm{d}} \in[0,1] . \eta_{\mathrm{d}}$ and $\eta$ are the joint vectors of the desired position and actual position, respectively.

Based on the PID sliding manifold of Equation (13) and sliding mode control theory, the sliding mode control law of the manipulator will be designed as Equation (14).

$$
U=k_{d}^{-1}\left[k_{p} \dot{e}+k_{i} e+k_{d} \ddot{x}_{d}+k_{d} \hat{H}+h s+\rho \operatorname{sign}(s)\right]+u_{c}
$$

where, $h$ is the positive constant, $\ddot{x}_{d}=\ddot{\eta}_{d}$, and $u_{\mathrm{c}}$ is the robust term of sliding mode control. In Equation (14), it is difficult to select a reasonable value for the error-boundary $\rho$, too conservative values would cause high frequency chattering of the system. To this end, the author obtains the error-boundary control law through the stability analysis, and adjusts the value of $\rho$ online, as shown in Equation (20).

Next, the first-order derivation of the sliding manifold (13a) with respect to time is used for the stability analysis. Then, substituting Equations (4) and (14) into (13a), we can get Equation (15). 


$$
\begin{aligned}
\dot{s} & =k_{p} \dot{e}+k_{i} e+k_{d} \ddot{x}_{d}-k_{d} U+k_{d} H \\
& =k_{d}(H-\hat{H})-[h s+\rho \operatorname{sign}(s)]-k_{d} u_{c}
\end{aligned}
$$

Substituting Equation (14) into Equation (15) and transforming it into an error state space equation, as shown in Equation (16).

$$
\ddot{e}+\left(k_{p} / k_{d}\right) \dot{e}+\left(k_{i} / k_{d}\right) e=(H-\hat{H})-k_{d}^{-1}[h s+\hat{\rho} \operatorname{sign}(s)]-u_{c}
$$

Converting Equation (16) to a matrix form:

$$
\begin{gathered}
\dot{E}=A E+B w \\
E=[e \dot{e}]^{T} A=\left[\begin{array}{cc}
0 & 1 \\
-k_{p} / k_{d} & -k_{i} / k_{d}
\end{array}\right]^{T} B=\left[\begin{array}{l}
0 \\
1
\end{array}\right] \\
w=(H-\hat{H})-k_{d}^{-1}[h s+\hat{\rho} \operatorname{sign}(s)]-u_{c}
\end{gathered}
$$

3.2.2. Adaptive Sliding Mode PID Control Law and Error-Boundary Control Law

Based on the above nonlinear sliding mode manifold, the main result of this paper is presented as follows:

Using the output of the Legendre polynomial FAT for control law (14), the adaptive sliding mode PID control law of this method in Equation (18) can be obtained. At the same time, the adaptive law of approximation coefficient $P$ and the error-boundary control law are shown in Equations (19) and (20), respectively.

$$
\begin{gathered}
U=k_{d}{ }^{-1}\left[k_{p} \dot{e}+k_{i} e+k_{d} \ddot{x}_{d}+k_{d} \hat{H}+h s+\hat{\rho}\left(K_{1} s+K_{2}|s|^{\alpha} \operatorname{sig}(s)\right)\right]+u_{c} \\
u_{c}=-k_{d}^{-1} h s^{T} \operatorname{sign}(s) \\
\hat{H}=\hat{P}^{T} \varphi(x) \\
\dot{\hat{P}}=\lambda_{1}\left[\varphi(x) s^{T} k_{d}+\varphi(x)\left(P_{0} B\right)^{T} E\right] \\
\dot{\hat{\rho}}=\lambda_{2}\left[s+k_{d}{ }^{-1}\left(E^{T} P_{0} B\right)^{T}\right]\left(K_{1} s+K_{2}|s|^{\rho} \operatorname{sig}(s)\right)
\end{gathered}
$$

where, $\lambda_{1}$ and $\lambda_{2}$ are the positive constant. $P_{0}$ and $Q$ are the positive definite matrices, so that $A^{T} P_{0}+P_{0} A=-Q$. In Equations (18a) and (20), the discontinuous switching term sign(s) in Equation (14) is replaced by $K_{1} s+K_{2}|s|^{\alpha} \operatorname{sig}(s)$ [27] to reduce the chattering of control voltage.

Based on the above control law, the control system block diagram of the proposed controller in this article is shown in Figure 1. 


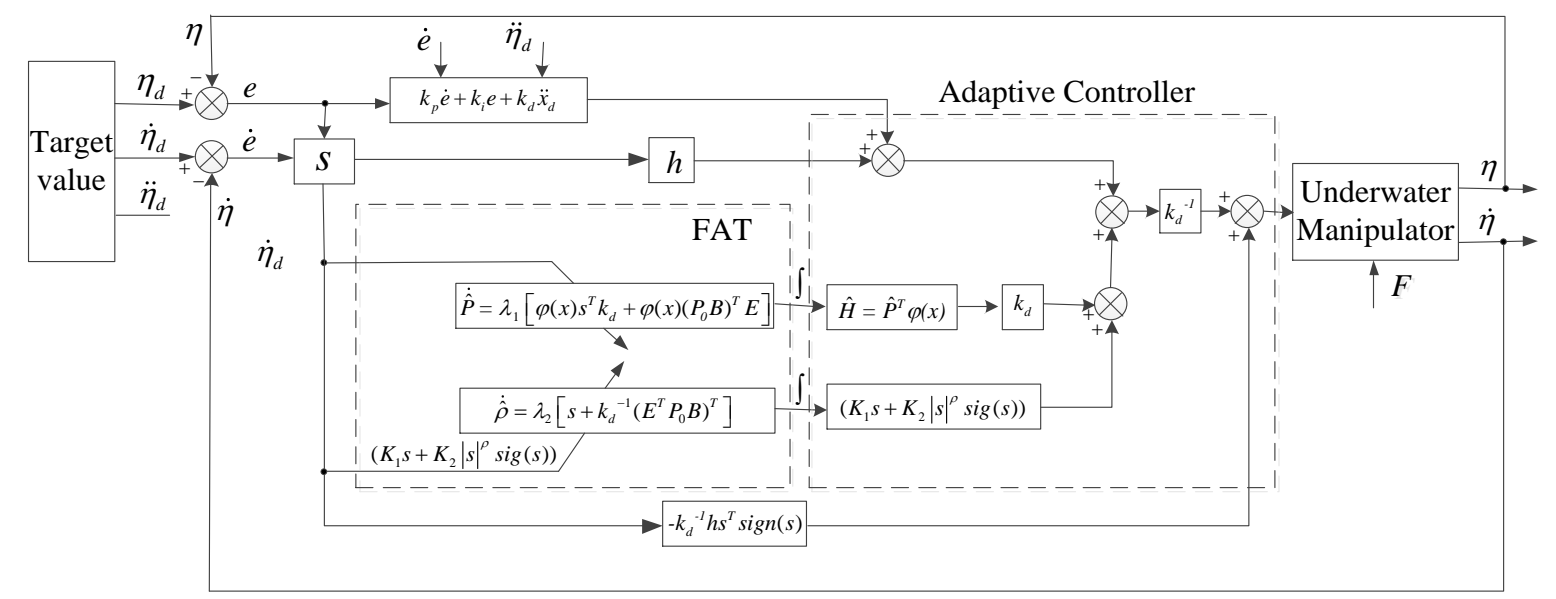

Figure 1. The control system block diagram of the proposed controller.

\subsection{Stability Analysis of the Proposed Method}

For the adaptive control law and error-boundary control law in Equations (18)-(20), the Lyapunov theory is used for the stability analysis to prove the boundedness of control parameters and the uniformly convergence of the joint error.

The following lemma is needed for the stability analysis.

Lemma 1. [26] If the differentiable function $\Delta(t)$ has a finite limit as $t \rightarrow+\infty$, and if $\dot{\Delta}(t)$ is uniformly continuous with respect to $t$ (a sufficient condition for a differentiable function to be uniformly continuous is that its derivative is bounded), then $\dot{\Delta}(t) \rightarrow 0$ as $t \rightarrow \infty$.

Consider the following Lyapunov function candidate:

$$
V=\frac{1}{2} s^{T} S+\frac{1}{2} E^{T} P_{0} E+\frac{1}{2 \lambda_{1}} \widetilde{P}^{T} \widetilde{P}+\frac{1}{2 \lambda_{2}} \widetilde{\rho}^{T} \widetilde{\rho}
$$

Combined Equation (17), the time derivative of Equation (21) is

$$
\begin{aligned}
\dot{V} & =s^{T} \dot{\mathcal{S}}+\frac{1}{2} \dot{E}^{T} P_{0} E+\frac{1}{2} E^{T} P_{0} \dot{E}+\frac{1}{\lambda_{1}} \widetilde{P}^{T} \dot{\widetilde{P}}+\frac{1}{\lambda_{2}} \widetilde{\rho}^{T} \dot{\tilde{\rho}} \\
& =s^{T} \dot{S}+\frac{1}{2} E^{T} A^{T} P_{0} E+\frac{1}{2} w^{T} B^{T} P_{0} E+\frac{1}{2} E^{T} P_{0} A E+\frac{1}{2} E^{T} P_{0} B w-\frac{1}{\lambda_{1}} \widetilde{P}^{T} \dot{\hat{P}}-\frac{1}{\lambda_{2}} \widetilde{\rho}^{T} \dot{\hat{\rho}} \\
& =s^{T} \dot{\mathcal{S}}-\frac{1}{2} E^{T} Q E+E^{T} P_{0} B w-\frac{1}{\lambda_{1}} \widetilde{P}^{T} \dot{\hat{P}}-\frac{1}{\lambda_{2}} \widetilde{\rho}^{T} \dot{\hat{\rho}}
\end{aligned}
$$

Substituting Equation (15) into $s^{T} \dot{s}$ in the Equation (22), and then integrating the Equations (11) and (12), it can yield

$$
\begin{aligned}
s^{T} \dot{s} & =s^{T} k_{d}(H-\hat{H})-s^{T}[h s+\hat{\rho} \operatorname{sign}(s)]-s^{T} k_{d} u_{c} \\
& =s^{T} k_{d} \widetilde{P}^{T} \varphi(x)-s^{T} h s-s^{T} \hat{\rho} \operatorname{sign}(s)-s^{T} k_{d} u_{c}+s^{T} k_{d} \varepsilon_{m}
\end{aligned}
$$

Substituting Equations (17c), (11) and (12) into $E^{T} P_{0} B w$ in the Equation (22), one has

$$
E^{T} P_{0} B w=E^{T} P_{0} B \widetilde{P}^{T} \varphi(x)-E^{T} P_{0} B k_{d}^{-1}[h s+\hat{\rho} \operatorname{sign}(s)]-E^{T} P_{0} B u_{c}+E^{T} P_{0} B e_{m}
$$

Then, in a similar way, substituting Equations (23) and (24) into Equation (25), we have

$$
\dot{V}=-\frac{1}{2} E^{T} Q E+V_{1}+V_{2}
$$




$$
\begin{gathered}
V_{1}=\widetilde{P}^{T}\left[s^{T} k_{d} \varphi(x)+E^{T} P_{0} B \varphi(x)\right]-\frac{1}{\lambda_{1}} \widetilde{P}^{T} \dot{\hat{P}}-\left[s^{T} k_{d}+E^{T} P_{0} B\right]\left[u_{c}+k_{d}{ }^{-1} h s\right] \\
V_{2}=\left[s^{T} k_{d} \varepsilon_{m}-s^{T} \hat{\rho} \operatorname{sign}(s)\right]+\left[E^{T} P_{0} B \varepsilon_{m}-E^{T} P_{0} B k_{d}{ }^{-1} \hat{\rho} \operatorname{sign}(s)\right]-\frac{1}{\lambda_{2}} \widetilde{\rho}^{T} \hat{\rho}
\end{gathered}
$$

On the basis of Equation (18b) and adaptive law Equation (19), we obtain $V_{1}<0$.

Due to $\widetilde{\rho}=\rho-\hat{\rho}$, the Equation (25c) can be expressed as

$$
\begin{aligned}
V_{2}= & {\left[s^{T} k_{d} \varepsilon_{m}-s^{T} \rho \operatorname{sign}(s)\right]+\left[E^{T} P_{0} B \varepsilon_{m}-E^{T} P_{0} B k_{d}^{-1} \rho \operatorname{sign}(s)\right] } \\
& +\widetilde{\rho}^{T}\left[s+k_{d}^{-1}\left(E^{T} P_{0} B\right)^{T}\right] \operatorname{sign}(s)-\frac{1}{\lambda_{2}} \widetilde{\rho}^{T} \hat{\rho}
\end{aligned}
$$

Thus, according to the error-boundary control law Equation (20), yields

$$
V_{2}=\left[s^{T} k_{d} \varepsilon_{m}-s^{T} \rho \operatorname{sign}(s)\right]+\left[E^{T} P_{0} B \varepsilon_{m}-E^{T} P_{0} B k_{d}^{-1} \rho \operatorname{sign}(s)\right]
$$

For (27), to prove the stability of the control system (i.e., $\dot{V} \leq 0$ ), the necessary and sufficient conditions are as follows.

$$
\begin{gathered}
s^{T} k_{d} \varepsilon_{m}-s^{T} \rho \operatorname{sign}(s) \leq 0 \\
E^{T} P_{0} B \varepsilon_{m}-E^{T} P_{0} B k_{d}^{-1} \rho \operatorname{sign}(s) \leq 0
\end{gathered}
$$

Therefore, to satisfy the above formula, the following analysis proves

(a) Due to $k_{d} \in[0,1]$, we have $\left|\varepsilon_{m}\right| \leq \rho \leq \delta=\rho / k_{\mathrm{d}}$, then

$$
\begin{aligned}
s^{T} k_{d} \varepsilon_{m}-s^{T} \rho \operatorname{sign}(s) & =k_{d}\left[s^{T} \varepsilon_{m}-s^{T} \delta \operatorname{sign}(s)\right] \\
& \leq k_{d}|s|\left(\left|\varepsilon_{m}\right|-\delta\right) \leq 0
\end{aligned}
$$

(b) From the sliding mode manifold $s, E=[e \dot{e}]^{T}$ and positive definite matrices $P_{0}$, it indicates that $\operatorname{sign}(s)=\operatorname{sign}\left(E^{\mathrm{T}} P_{0} \mathrm{~B}\right)$ and we can be rewritten as

$$
\begin{aligned}
& E^{T} P_{0} B \varepsilon_{m}-E^{T} P_{0} B k_{d}^{-1} \rho \operatorname{sign}(s)=E^{T} P_{0} B \varepsilon_{m}-E^{T} P_{0} B k_{d}{ }^{-1} \rho \operatorname{sign}\left(E^{T} P_{0} B\right) \\
& \leq\left|E^{T} P_{0} B\right|\left(\left|\varepsilon_{m}\right|-k_{d}^{-1} \rho\right) \\
& \leq\left|E^{T} P_{0} B\right|\left(\left|\varepsilon_{m}\right|-\delta\right) \leq 0
\end{aligned}
$$

According to the above proof (i.e., (a) and (b)), it can be obtain that $V_{2} \leq 0$. Meanwhile, from the Equations (27)-(30), yields

$$
\dot{V}=-\frac{1}{2} E^{T} Q E+V_{1}+V_{2} \leq-\frac{1}{2} E^{T} Q E
$$

By integrating inequality Equation (31), it indicates $\dot{V} \leq 0$, and then we can get $s, E, P$, and $\rho$ are bounded. Then, it will prove that $s$ and $E$ will be uniformly asymptotically convergence.

Since $\dot{V} \leq 0$ is semi-negative, let $\Delta(t)=-\frac{1}{2} E^{T} Q E$. Then, integrating inequality Equation (31) on the interval $[0, t]$, it can obtained that $\int_{0}^{t} \Delta(t) d t \leq V(0)$ is bounded, this indicates $\int_{0}^{t} \Delta(t) d t$ has a finite limit, as $t \rightarrow+\infty$. Due to $E$ is bounded, so it has that $\dot{E}$ is bounded, and further conclude $\Delta(t)$ is bounded. Then, it can be obtained that $\Delta(t)$ is continuous with respect to $t$. Based on Lemma 1, we can have $\Delta(t) \rightarrow 0$ as $t \rightarrow \infty$, which implies that $s$ and $E$ are equal to zero. As a result, it can be proved that the tracking error $e$ and $s$ are uniformly asymptotic converged and stable.

\section{Pool-Experiments Verification and Discussion}

In this section, the effectiveness of the proposed methods (i.e., adaptive sliding mode PID control based on FAT) is validated on the underwater manipulator of the UVIC-I AUV, and in the pool-environment, the experimental results of the proposed methods are compared with the method 
in [17]. The UVIC-I AUV, as shown in Figure 2a, is a prototype vehicle designed for underwater operations, mainly consisting of AUVs and underwater manipulator.

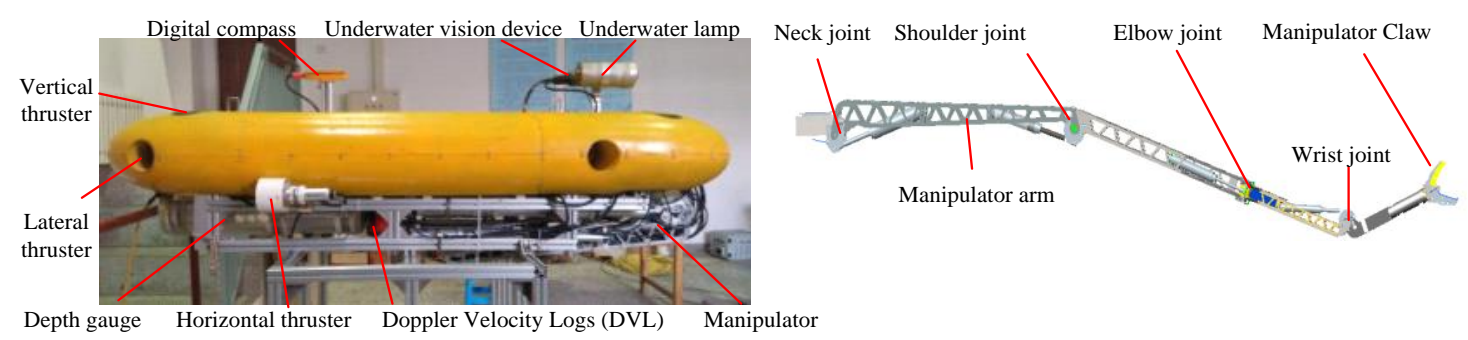

(a)

(b)

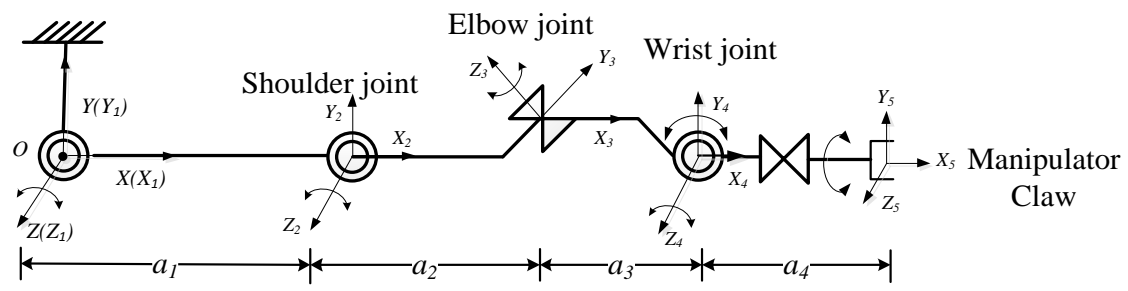

(c)

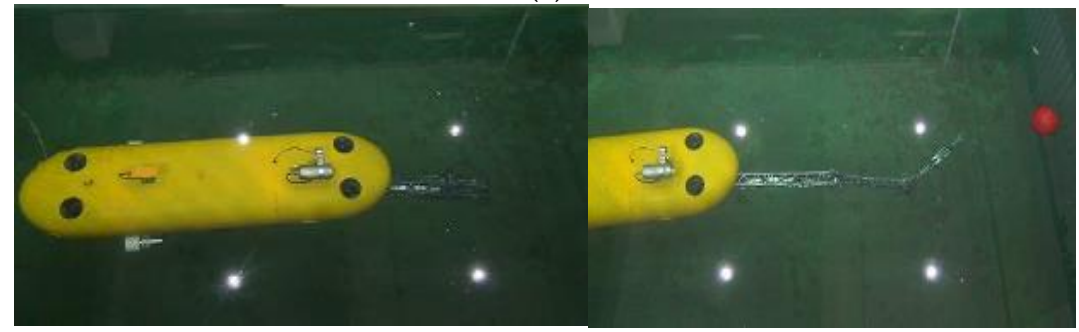

(d)

Figure 2. UVIC-I autonomous underwater vehicle (AUV) and its underwater operations. (a) The UVIC-I prototype vehicle; (b) overall structure of underwater manipulator; (c) mechanism diagram of the underwater manipulator; and (d) UVIC-I AUV during underwater operations.

The AUVs is equipped with eight thrusters, two to actuate surge, two to actuate sway and yaw, and four vertical thrusters to actuate heave, roll, and pitch, and sensors such as depth, velocity, and attitude angle [28]. The underwater manipulator, as shown in Figure $2 b, c$, with five joints (5 DOF), the detailed structure is shown in [9], and the main parameters are shown in Table 1. Moreover, during the underwater experiments, the experiments are obtained in a pool measuring $8.0 \mathrm{~m} \times 5.0 \mathrm{~m} \times 5.0 \mathrm{~m}$, and the AUVs is in a dynamic positioning state, maintaining a stable attitude, as shown in Figure $2 \mathrm{~d}$. The main parameters of the adaptive sliding mode PID controller are given as follows: $\omega=0.2, k_{\mathrm{p}}=$ $0.5, k_{\mathrm{i}}=0.1, k_{\mathrm{d}}=1, h=1, \lambda_{1}=10, \lambda_{2}=1, A=[01 ; 0.50 .1], Q=[4000 ; 0400], K_{1}=1.5$.

Table 1. The main parameters of the underwater manipulator.

\begin{tabular}{cccc}
\hline & Length & Weight & Rotation Angle Range of Each Joint \\
\hline Neck joint & 0 & $5.5 \mathrm{~kg}$ & 0 to $-180^{\circ}$ \\
Shoulder joint & $900 \mathrm{~mm}\left(a_{1}\right)$ & $2.0 \mathrm{~kg}$ & 0 to $-180^{\circ}$ \\
Elbow joint & $600 \mathrm{~mm}\left(a_{2}\right)$ & $1.5 \mathrm{~kg}$ & -30 to $180^{\circ}$ \\
Wrist joint & $400 \mathrm{~mm}\left(a_{3}\right)$ & $1.0 \mathrm{~kg}$ & 90 to $270^{\circ}$ \\
Manipulator Claw & $480 \mathrm{~mm}\left(a_{4}\right)$ & $1.0 \mathrm{~kg}$ & 0 to $180^{\circ}$ \\
\hline
\end{tabular}

\subsection{The Joint Control Pool-Experiments of the Underwater Manipulator}

The proposed methods in this paper mainly focused on improving the joint control accuracy of the manipulator, and reducing the influences of joint lag and the trajectory tracking error. Therefore, 
compared with the method in [17], the joint step response experiments and trajectory tracking experiments were designed to verify the effectiveness of the proposed method.

\subsubsection{Experiments of Joint Step Response}

For the experiments of the joint step response, the manipulator was fixed on the UVIC-I AUV and the each joints move from the initial angle to the target angle. The two sets of initial and target angles of (neck, shoulder, elbow, wrist) joints in this paper are considered as follows

1st. initial angle $\left(45.0^{\circ}, 80.0^{\circ}, 80.0^{\circ}, 60.0^{\circ}\right)$ and target angle $\left(115.0^{\circ}, 110.0^{\circ}, 35.0^{\circ}, 130.0^{\circ}\right)$.

2st. initial angle $\left(80.0^{\circ}, 120.0^{\circ}, 35.0^{\circ}, 100.0^{\circ}\right)$ and target angle $\left(8.0^{\circ}, 165.0^{\circ}, 150.0^{\circ}, 10.0^{\circ}\right)$.

The experiment results of the proposed method and the method in [17] are shown in Figure 3. For the convenience of analysis, the relevant indicator data in Figure 3, such as the overshoot, steady state error, is summarized in Tables 2 and 3.

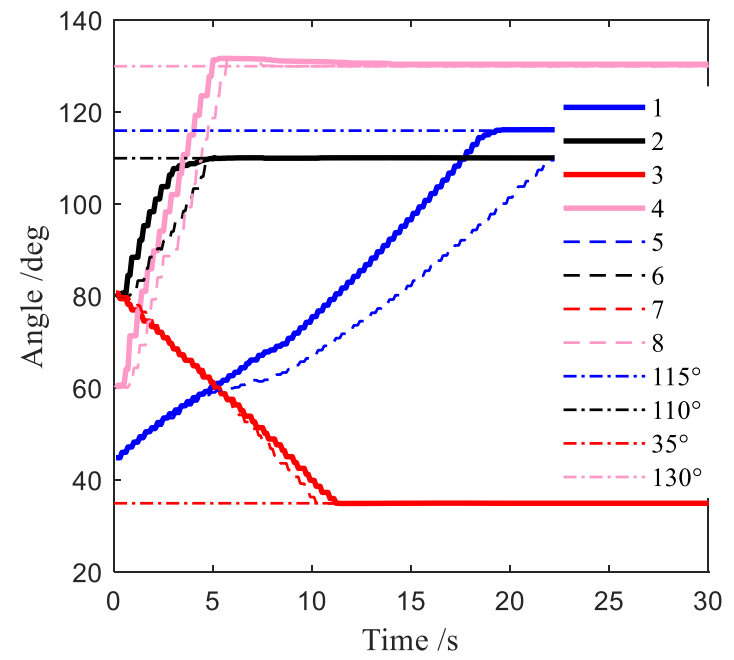

(a)

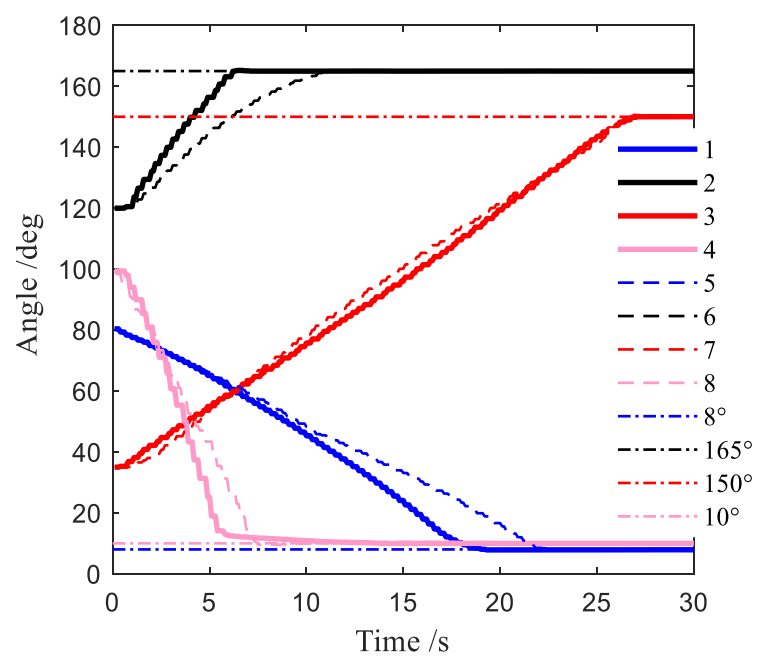

(b)

Figure 3. Step response curve of each joint of underwater manipulator. (a) Step response curve of 1st and (b) step response curve of 2st.

Table 2. Statistical Results of Figure 3a.

\begin{tabular}{ccc}
\hline & Overshoot & Steady State Error \\
\hline The proposed method & $(0.36,0.62,0.43,0.55)^{\circ}$ & $(0.025,0.017,0.017,0.025)^{\circ}$ \\
The method in [17] & $(0.68,1.02,0.98,1.38)^{\circ}$ & $(0.035,0.024,0.026,0.031)^{\circ}$ \\
Reduced by & $(47.06 \%, 39.22 \%, 56.12 \%, 60.14 \%)$ & $(28.57 \%, 29.17 \%, 34.62 \%, 19.35 \%)$ \\
\hline
\end{tabular}

Table 3. Statistical Results of Figure 3b.

\begin{tabular}{ccc}
\hline & Overshoot & Steady State Error \\
\hline The proposed method & $(0.32,0.51,0.39,0.58)^{\circ}$ & $(0.022,0.017,0.018,0.026)^{\circ}$ \\
The method in [17] & $(0.72,0.91,1.04,1.35)^{\circ}$ & $(0.030,0.022,0.025,0.031)^{\circ}$ \\
Reduced by & $(55.56 \%, 43.95 \%, 62.50 \%, 57.04 \%)$ & $(26.67 \%, 22.73 \%, 28.00 \%, 16.13 \%)$ \\
\hline
\end{tabular}

Note: 1, 2, 3, and 4 are the step response curves of (neck, shoulder, elbow, wrist) joints of the proposed method, respectively. The step response curves of (neck, shoulder, elbow, wrist) joints of the method in [17] are 5, 6, 7, and 8, respectively

From Figure 3, it can be seen that the angles of the joints were uniformly converged. In addition, compared with the method in [17], the joint angular velocity (i.e., to illustrate the response speed of system) in this paper had a certain increase. This is due to the fact that the PID sliding manifold in the 
paper could improve the system response speed of each joint of the manipulator. From the Tables 2 and 3 , it is shown that the proposed method greatly improved the overshoot and steady state error of the joint of the underwater manipulator. The above data show that the proposed method could effectively improve the joint control accuracy, and verified the effectiveness of the proposed method.

\subsubsection{Experiments of Joint Continuous Trajectory Tracking}

In the experiments of joint continuous trajectory tracking, consistent with the method in [17], the sinusoid was selected as the target trajectory. The target sinusoidal trajectory of the (neck, shoulder, elbow, wrist) joints is as shown in the following Equation (32).

$$
\eta=A \sin \left(\frac{2 \pi}{100} t\right)+B
$$

where, $\eta=\left(\eta_{1}, \eta_{2}, \eta_{3}, \eta_{4}\right)^{\mathrm{T}}, A=\left(50^{\circ}, 30^{\circ}, 50^{\circ}, 30^{\circ}\right)^{\mathrm{T}}, B=\left(90^{\circ}, 90^{\circ}, 120^{\circ}, 150^{\circ}\right)^{\mathrm{T}}$.

The pool-experiments results of the proposed method and the method in [17] are shown in Figure 4. Moreover, and the relevant indicator data in Figure 4 are summarized into Table 4 for a quantitative analysis. For the experimental analysis, the "Maximum absolute tracking error (MATE), Standard deviation of error (SDE)" was used as the evaluation index. At the same time, to illustrate the effect of the proposed method on reducing the joint lag, increase the "motion lag time" as the evaluation index.

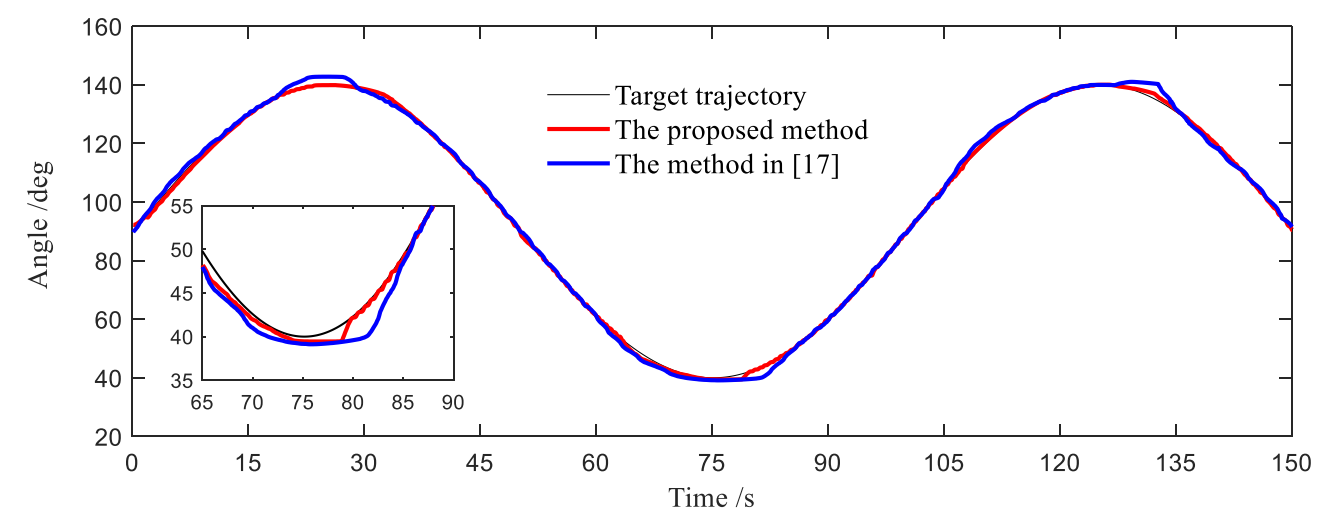

(a)

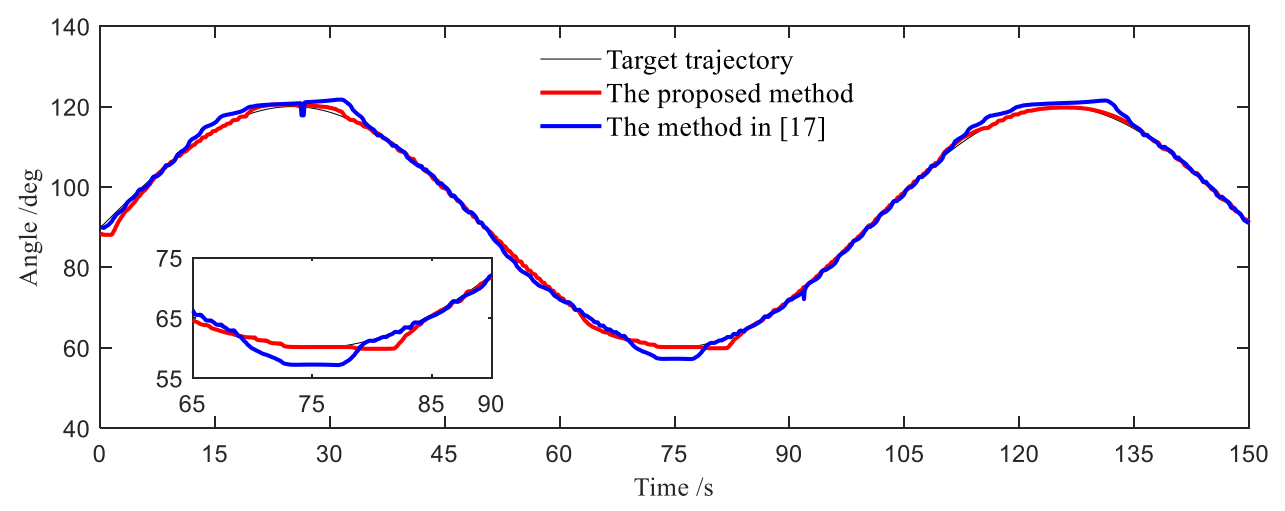

(b)

Figure 4. Cont. 


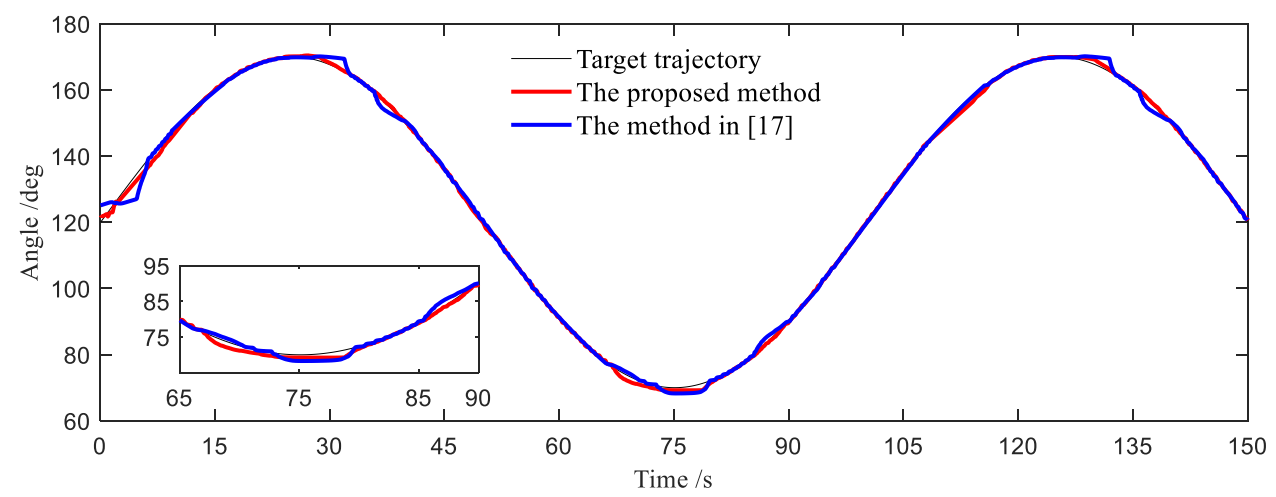

(c)

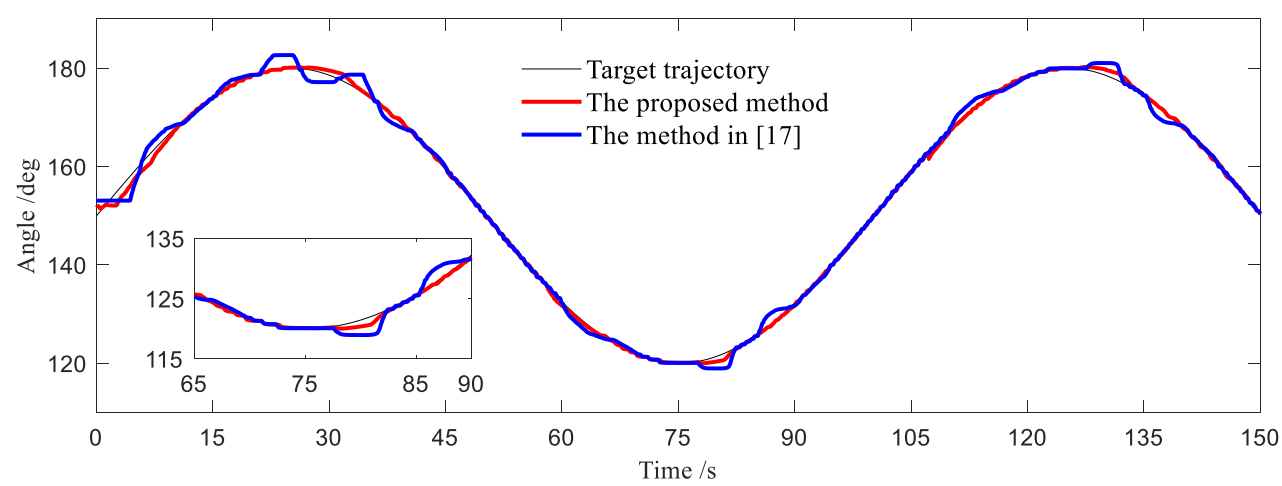

(d)

Figure 4. Joint trajectory tracking curve of each joint of underwater manipulator. (a) Neck joint; (b) shoulder joint; (c) elbow joint; and (d) wrist joint.

Table 4. Pool-experiment results of sinusoidal trajectory tracking.

\begin{tabular}{cccc}
\hline & MATE & SDE & Motion Lag Time \\
\hline The proposed method & $(1.971,2.716,2.660,2.247) \mathrm{deg}$ & $(0.999,1.149,1.159,0.896) \mathrm{deg}$ & $(3.0,2.9,4.4,3.2) \mathrm{s}$ \\
The method in [17] & $(3.086,3.826,3.942,3.678) \mathrm{deg}$ & $(1.152,1.340,1.462,1.333) \mathrm{deg}$ & $(3.9,4.1,5.5,4.7) \mathrm{s}$ \\
Reduced by & $(36.13 \%, 29.01 \%$, & $(13.28 \%, 14.25 \%$, & $(23.08 \%, 29.27 \%$, \\
& $32.52 \%, 38.91 \%)$ & $20.73 \%, 32.78 \%)$ & $20.00 \%, 31.91 \%)$ \\
\hline
\end{tabular}

From Table 4, in comparison with the method in [17], the results confirm that the proposed method can reduce the trajectory tracking error, and reduce the influence of joint lag on the joint control of underwater manipulator. The results verified the effectiveness of the proposed method.

\subsection{The Control Voltage of Underwater Manipulator}

In this section, the comparative experimental analysis of the joint control voltage of the proposed method and the method in [17] is carried out, to verify that the proposed method can reduce the chattering of control voltage.

Under this circumstance of continuous trajectory tracking experiments, the target trajectory is Equation (32). The contrast experimental results of the two methods are shown in Figure 5. 


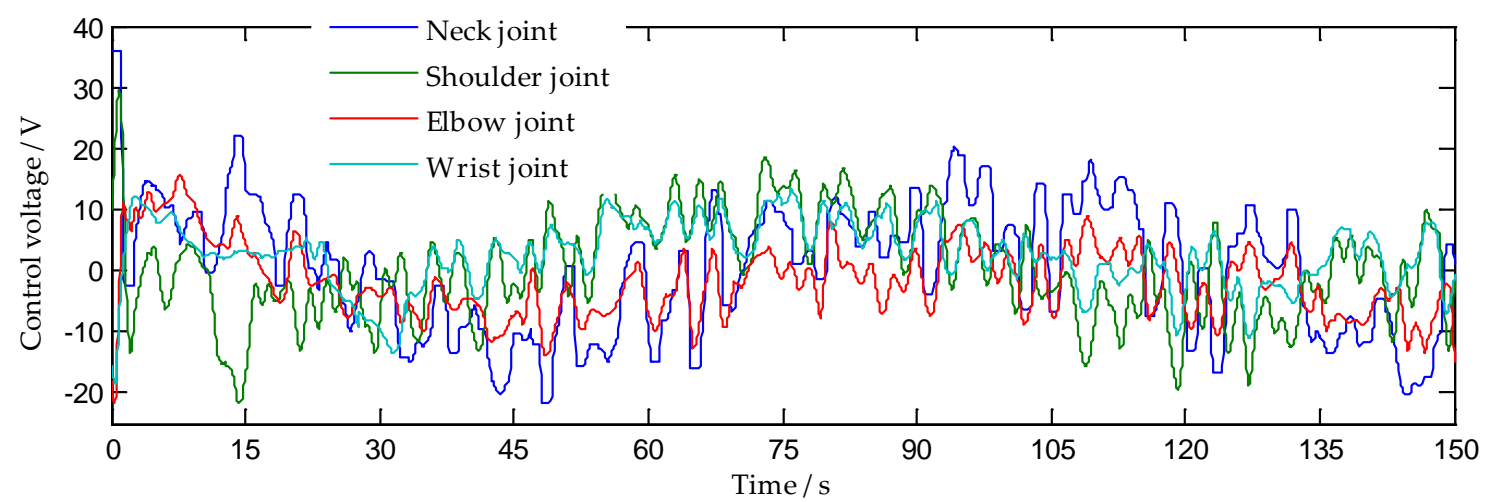

(a)

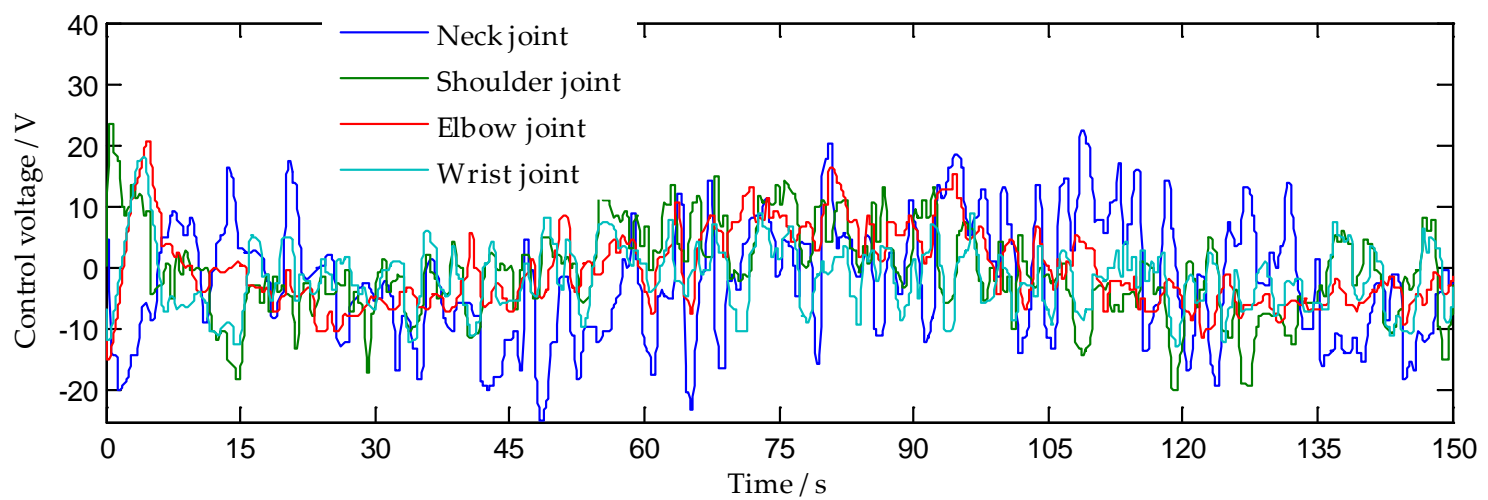

(b)

Figure 5. The comparison data of joint control voltages of underwater manipulators. (a) The control voltages of the proposed method and (b) the control voltages of the method in [17].

To give a quantitative analysis, the results of Figure 5 are summarized in Table 5. In addition, consistent with the reference [25], the standard deviation (SD) was used as the evaluation index for the chattering amplitude of the control voltage, and the standard deviation of the derivatives of control variables (SDDCV) was used to evaluate the chattering frequency of control voltage.

Table 5. Statistical result of Figure 5.

\begin{tabular}{ccc}
\hline & SD & SDDCV \\
\hline The proposed method & $(10.196,7.849,5.880,5.368) \mathrm{V}$ & $(0.671,0.446,0.309,0.250) \mathrm{V}$ \\
The method in [17] & $(9.908,7.415,6.358,5.440) \mathrm{V}$ & $(1.072,0.756,0.486,0.648) \mathrm{V}$ \\
Reduced by & $(-2.91 \%,-5.85 \%, 7.52 \%, 1.33 \%)$ & $(37.42 \%, 41.03 \%, 36.36 \%, 61.49 \%)$ \\
\hline
\end{tabular}

From Table 5, compared with the method in [17], the above results validated that the chattering amplitude of the control voltage was roughly the same as the method in [17], but the proposed method could greatly reduce the chattering frequency, which is beneficial to the protection of the motor of the underwater manipulator.

\section{Conclusions}

In this paper, a novel adaptive sliding mode PID control method based on Legendre polynomial FAT was addressed for the joint control of underwater manipulator, and the system error relying on this approach was proven to be uniformly asymptotic convergence. The adaptive sliding mode PID controller of the approach was designed to reduce the impact of joint lag, and adopted FAT to approximate uncertainty factors online for the control system. Moreover, it did not need to know the upper bound of any uncertainties and external disturbances. 
The experimental results provided very strong evidence that the proposed method could effectively improve the control accuracy of the manipulator joint, and reduced the influence of the joint lag on the trajectory tracking control. Meanwhile, it could reduce the frequency of the chattering of the control voltages, compared with the comparison method, the SDDCV of the proposed method was decreased $(37.42 \%, 41.03 \%, 36.36 \%, 61.49 \%)$. The effectiveness of the proposed method was verified by theoretical research and experiments. The research of this paper lays a foundation for the underwater operation of the UVIC-I AUV of the support project.

Author Contributions: Conceptualization and methodology, C.Y. and M.Z.; data curation, C.Y., Z.Z., Z.W. and P.D.; formal analysis, software and validation, C.Y. and F.Y.; project administration and resources, M.Z.; writing-original draft, C.Y. All authors have read and agreed to the published version of the manuscript.

Funding: This research was funded by the NATIONAL NATURAL SCIENCE FOUNDATION OF CHINA, grant number 51839004. The project was supported by CAEP foundation, grant No.PY20200104.

Acknowledgments: The authors thank the financial support from the NATIONAL NATURAL SCIENCE FOUNDATION OF CHINA with Grant number 51839004, and the project supported by CAEP foundation with Grant number PY20200104.

Conflicts of Interest: The authors declare no conflict of interest.

\section{References}

1. Shen, C.; Shi, Y.; Buckham, B. Trajectory Tracking Control of an Autonomous Underwater Vehicle using Lyapunov-based Model Predictive Control. IEEE Trans. Ind. Electron. 2018, 65, 5796-5805. [CrossRef]

2. Wang, R.; Wang, X.; Zhu, M.M.; Lin, Y.F. Application of a Real-Time Visualization Method of AUVs in Underwater Visual Localization. Appl. Sci. 2019, 9, 1428. [CrossRef]

3. Paull, L.; Saeedi, S.; Seto, M.; Li, H. AUV Navigation and Localization: A Review. IEEE J. Ocean. Eng. 2014, 39, 131-149. [CrossRef]

4. Simetti, E.; Casalino, G.; Torelli, S.; Sperindé, A.; Turetta, A. Floating Underwater Manipulation: Developed Control Methodology and Experimental Validation within the TRIDENT Project. J. Field. Rob. 2014, 31, 364-385. [CrossRef]

5. Marani, G.; Choi, S.K.; Yuh, J. Underwater autonomous manipulation for intervention missions AUVs. Ocean Eng. 2009, 36, 15-23. [CrossRef]

6. Luo, G.S. Research on Subsea 7 Function Master-Slave Hydraulic Manipulator and Its Nonlinear Robust Control. Ph.D. Thesis, Zhejiang University, Hangzhou, China, 2013.

7. Tran, M.D.; Kang, H.J. Adaptive terminal sliding mode control of uncertain robotic manipulators based on local approximation of a dynamic system. Neurocomputing 2017, 228, 231-240. [CrossRef]

8. Sivčev, S.; Coleman, J.; Omerdić, E.; Dooly, G.; Toal, D. Underwater manipulators: A review. Ocean Eng. 2018, 163, 431-450. [CrossRef]

9. Yang, C.; Wang, Y.J.; Yao, F. Driving performance of underwater long-arm hydraulic manipulator system for small autonomous underwater vehicle and its positioning accuracy. Int. J. Adv. Rob. Syst. 2017, 14. [CrossRef]

10. Zhang, J.; Li, W.; Yu, J.C.; Li, Y. Virtual platform of a manned submersible vehicle carrying an underwater manipulator. In Proceedings of the 2015 IEEE International Conference on Cyber Technology in Automation, Control, and Intelligent Systems, Shenyang, China, 8-12 June 2015; pp. 1126-1131.

11. Hacioglu, Y.; Arslan, Y.Z.; Yagiz, N. MIMO fuzzy sliding mode controlled dual arm robot in load transportation. J. Franklin Inst. 2011, 348, 1886-1902. [CrossRef]

12. Shafiei, S.E.; Soltanpour, M.R. Robust Neural Network Control of Electrically Driven Robot Manipulator using Backstepping Approach. Int. J. Adv. Robot. Syst. 2010, 6, 285-292. [CrossRef]

13. Kumar, N.; Panwar, V.; Sukavanam, N.; Sharma, S.P.; Borma, J.H. Neural network-based nonlinear tracking control of kinematically redundant robot manipulators. Math. Comput. Model. 2011, 53, 1889-1901. [CrossRef]

14. Fateh, M.M.; Khorashadizadeh, S. Robust control of electrically driven robots by adaptive fuzzy estimation of uncertainty. Nonlinear Dyn. 2012, 69, 1465-1477. [CrossRef]

15. Rodrigo, H.A.; Govinda, G.V.L.; Tomás, S.J.; Alfonso, G.E.; Fernando, F.N. Neural network-based self-tuning PID control for underwater vehicles. Sensors 2016, 16, 1429. 
16. Zhang, M.J.; Chu, Z.Z. Adaptive sliding mode control based on local recurrent neural networks for underwater robot. Ocean Eng. 2012, 45, 56-62. [CrossRef]

17. Zirkohi, M.M. Direct Adaptive Function Approximation Techniques Based Control of Robot Manipulators. J. Dyn. Syst., Meas., Control 2018, 140, 011006. [CrossRef]

18. Ahmadi, S.M.; Fateh, M.M. Robust control of electrically driven robots using adaptive uncertainty estimation. Comput. Electr. Eng. 2016, 56, 674-687. [CrossRef]

19. Khorashadizadeh, S.; Fateh, M.M. Adaptive fourier series-based control of electrically driven robot manipulators. In Proceedings of the International Conference on Control, Instrumentation, and Automation, Tehran, Iran, 28-30 December 2013; pp. 213-218.

20. Khorashadizadeh, S.; Fateh, M.M. Robust task-space control of robot manipulators using Legendre polynomials for uncertainty estimation. Nonlinear Dyn. 2014, 79, 1151-1161. [CrossRef]

21. Javier, M.V.; Carlos, T.T. Adaptive chaotification of robot manipulators via neural networks with experimental evaluations. Neurocomputing 2016, 182, 56-65.

22. Zhang, M.; Chu, Z. Research on generic model control method for manipulator based on neural networks. In Proceedings of the 2012 IEEE International Conference on Mechatronics and Automation, Chengdu, China, 5-8 August 2012; pp. 371-376.

23. Wang, Y.J.; Zhang, M.J.; Chu, Z.Z.; Liu, X. Fault-tolerant control based on adaptive sliding mode for underwater vehicle with thruster fault. In Proceedings of the 11th World Congress on Intelligent Control and Automation, Shenyang, China, 29 June-4 July 2014; pp. 5323-5328.

24. Kreyszig, E. Advanced Engineering Mathematics: Maple Computer Guide; John Wiley \& Sons: New York, NY, USA, 2000.

25. Wang, Y.; Zhang, M.J.; Wilson, P.A.; Liu, X. Adaptive neural network-based backstepping fault tolerant control for underwater vehicles with thruster fault. Ocean Eng. 2015, 110, 15-24. [CrossRef]

26. Zhang, M.; Liu, X.; Yin, B.; Liu, W.X. Adaptive terminal sliding mode based thruster fault tolerant control for underwater vehicle in time-varying ocean currents. J. Franklin Inst. 2015, 352, 4935-4961. [CrossRef]

27. Yang, C.; Yao, F.; Zhang, M. Adaptive Backstepping Terminal Sliding Mode Control Method Based on Recurrent Neural Networks for Autonomous Underwater Vehicle. Chin. J. Mech. Eng. 2018, 31, 110. [CrossRef]

28. Feng, Y.; Chao, Y.; Xing, L.; Zhang, M.J. Experimental Evaluation on Depth Control Using Improved Model Predictive Control for Autonomous Underwater Vehicle (AUVs). Sensors 2018, 18, 2321. 